\title{
Research Article \\ Output Feedback Stabilization of Linear Time-Varying Uncertain Delay Systems
}

\author{
Tomoaki Hashimoto $^{1}$ and Takashi Amemiya ${ }^{2}$ \\ ${ }^{1}$ Department of Electrical and Electronic Engineering, Shinshu University, 4-17-1 Wakasato, \\ Nagano 380-8553, Japan \\ ${ }^{2}$ Department of Business Administration and Information, Setsunan University, 17-8 Ikeda-naka-machi, \\ Neyagawa, Osaka 572-8508, Japan
}

Correspondence should be addressed to Tomoaki Hashimoto, info@thashi.net

Received 27 February 2009; Accepted 24 May 2009

Recommended by John Burns

This paper investigates the output feedback stabilization problem of linear time-varying uncertain delay systems with limited measurable state variables. Each uncertain parameter and each delay under consideration may take arbitrarily large values. In such a situation, the locations of uncertain entries in the system matrices play an important role. It has been shown that if a system has a particular configuration called a triangular configuration, then the system is stabilizable irrespective of the given bounds of uncertain variations. In the results so far obtained, the stabilization problem has been reduced to finding the proper variable transformation such that an $M$-matrix stability criterion is satisfied. However, it still has not been shown whether the constructed variable transformation enables the system to satisfy the $M$-matrix stability condition. The objective of this paper is to show a method that enables verification of whether the transformed system satisfies the $M$-matrix stability condition.

Copyright (c) 2009 T. Hashimoto and T. Amemiya. This is an open access article distributed under the Creative Commons Attribution License, which permits unrestricted use, distribution, and reproduction in any medium, provided the original work is properly cited.

\section{Introduction}

This paper examines the stabilization problem of linear time-varying uncertain delay systems by means of linear memoryless state feedback control. The systems under consideration contain uncertain entries in the system matrices and uncertain delays in the state variables. Each value of uncertain entries and delays may vary with time independently in an arbitrarily large bound. Under this situation, the locations of uncertain entries in the system matrices play an important role. This paper presents investigation of the permissible locations of uncertain entries, which are allowed to take unlimited large values, for the stabilization using linear state feedback control.

It is useful to classify the existing results on the stabilization of uncertain systems into two categories. The first category includes several results which provide the stabilizability conditions depending on the bounds of uncertain parameters. The results in the second 
category provide the stabilizability conditions that are independent of the bounds of uncertain parameters but which depend on their locations. This paper specifically addresses the second category.

For uncertain systems with delays, the Lyapunov stability approach with the Krasovskii-based or Razumikhin-based method is a commonly used tool. The stabilization problem has been reduced to solving linear matrix inequalities (LMIs) [1-3]. However, LMI conditions fall into the first category; for this reason, they are often used to determine the permissible bounds of uncertain parameters for the stabilization. When the bounds of uncertain parameter values exceed a certain value, LMI solver becomes infeasible. In such cases, guidelines for redesigning the controller are usually lacking.

On the other hand, the stabilizability conditions in the second category can be verified easily merely by examining the uncertainty locations in given system matrices. Once a system satisfies the stabilizability conditions, a stabilizing controller can be constructed, irrespective of the given bounds of uncertain variations. We can redesign the controller for improving robustness merely by modifying the design parameter when the uncertain parameters exceed the upper bounds given beforehand.

In the second category, the stabilization problem of linear time-varying uncertain systems without delays was studied by Wei [4]. The stabilizability conditions have a particular geometric configuration with respect to the permissible locations of uncertain entries. Using the concept of antisymmetric stepwise configuration (ASC) [4], Wei proved that a linear time-varying uncertain system is stabilizable independently of the given bounds of uncertain variations using linear state feedback control if and only if the system has an ASC. Wei derived the successful result on the stabilization problem of systems without delays, however, his method [4] is inapplicable to systems that contain delays in the state variables.

On the one hand, based on the properties of an M-matrix, Amemiya and Leitmann [5] developed the conditions for the stabilization of linear time-varying uncertain systems with time-varying delays using linear memoryless state feedback control. The conditions obtained in [5] show a similar configuration to an ASC, but the allowable uncertainty locations are fewer than in an ASC by one step.

The aforementioned results presume that all state variables are accessible for designing a controller. However, it is usual that the state variables of the systems are measured through the outputs and hence only limited parts of them can be used directly. The output feedback stabilization of linear uncertain delay systems with limited measurable state variables has been investigated in $[6,7]$. The conditions so far obtained show that if a system has a particular configuration called a triangular configuration, then the system is stabilizable independently of the given bounds of uncertain variations. The conditions in [7] consist of not only the system matrix $\Delta A$ but also input and output coefficient matrices, $\Delta B$ and $\Delta C$, while the conditions in [6] consist of only $\Delta A$.

The results so far obtained were derived using an $M$-matrix stability criterion. In [5-7], the stabilization problem has been reduced to finding the variable transformation such that the $M$-matrix stability condition is satisfied. Although the developed conditions called a triangular configuration has been shown in [7], it still has not been shown whether the constructed variable transformation enables the system to satisfy the $M$-matrix stability condition. The objective of this paper is to show a method that enables verification of whether the transformed system satisfies the $M$-matrix condition. This paper specially examines the functional-order structure of the transformed system in order to verify whether the system has an $M$-matrix structure. 
This paper is organized as follows. Some notations and terminology are given in Section 2. The systems considered here are defined in Section 3. In Section 4, some preliminary results are introduced to state the present problem. The main results are provided in Section 5. Finally, some concluding remarks are presented in Section 6.

\section{Notations and Terminology}

First, some notations and terminology used in the subsequent description are given. For $a, b \in$ $\mathbb{R}^{m}$ or $A, B \in \mathbb{R}^{n \times m}$, every inequality between $a$ and $b$ or $A$ and $B$ such as $a>b$ or $A>B$ indicates that it is satisfied component-wise by $a$ and $b$ or $A$ and $B$. If $A \in \mathbb{R}^{n \times m}$ satisfies $A \geq 0, A$ is called a nonnegative matrix. The transpose of $A \in \mathbb{R}^{n \times m}$ is denoted by $A^{\prime}$. For $a=\left(a_{1}, \ldots, a_{m}\right)^{\prime} \in \mathbb{R}^{m},|a| \in \mathbb{R}^{m}$ is defined as $|a|=\left(\left|a_{1}\right|, \ldots,\left|a_{m}\right|\right)^{\prime}$. Also for $A=\left(a_{i j}\right) \in \mathbb{R}^{n \times m}$, $|A|$ denotes a matrix with $\left|a_{i j}\right|$ as its $(i, j)$ entries. Let $\operatorname{diag}\{\cdots\}$ denote a diagonal matrix. Let $[a, b], a, b \in \mathbb{R}$ be an interval in $\mathbb{R}$. The set of all continuous or piecewise continuous functions with domain $[a, b]$ and range $\mathbb{R}^{n}$ is denoted, respectively, by $\mathcal{C}^{n}[a, b]$ or $\Phi^{n}[a, b]$. We denote it simply by $\mathcal{C}^{n}$ or $\mathbb{\Phi}^{n}$ if the domain is $\mathbb{R}$.

The notation for a class of functions is introduced below. Let $\xi(\mu) \in \mathcal{C}^{1}$ and let $m \in \mathbb{R}$ be a constant. If $\xi(\mu)$ satisfies the conditions

$$
\begin{aligned}
& \underset{|\mu| \rightarrow \infty}{\limsup }\left|\frac{\xi(\mu)}{\mu^{m}}\right|<\infty, \\
& \underset{|\mu| \rightarrow \infty}{\limsup }\left|\frac{\xi(\mu)}{\mu^{m-a}}\right|=\infty
\end{aligned}
$$

for any positive scalar $a \in \mathbb{R}$, then $\xi(\mu)$ is called a function of order $m$, and we denote this as follows:

$$
\operatorname{Ord}(\xi(\mu))=m
$$

The set of all $\mathcal{C}^{1}$ functions of order $m$ is denoted by $O(m)$,

$$
O(m)=\left\{\xi(\mu) \mid \xi(\mu) \in \mathcal{C}^{1}, \operatorname{Ord}(\xi(\mu))=m\right\}
$$

Also, it is worth to note that $m$ can be a negative number and that the following relations between $\xi_{1}(\mu) \in O\left(m_{1}\right)$ and $\xi_{2}(\mu) \in O\left(m_{2}\right)$ hold:

$$
\begin{gathered}
\operatorname{Ord}\left(\xi_{1}(\mu) \pm \xi_{2}(\mu)\right)=\max \left\{m_{1}, m_{2}\right\} \\
\operatorname{Ord}\left(\xi_{1}(\mu) \times \xi_{2}(\mu)\right)=m_{1}+m_{2} \\
\operatorname{Ord}\left(\frac{\xi_{1}(\mu)}{\xi_{2}(\mu)}\right)=m_{1}-m_{2}
\end{gathered}
$$


A real square matrix all of whose off-diagonal entries are nonpositive is called an $M$-matrix if it is nonsingular and its inverse matrix is nonnegative. The set of all $M$-matrices is denoted by $\mathcal{M}$.

\section{System Description}

Let $n$ be a fixed positive integer. The system considered here is given by a delay differential equation defined on $x \in \mathbb{R}^{n}$ for $t \in\left[t_{0}, \infty\right)$ as follows:

$$
\begin{gathered}
\dot{x}(t)=A^{0} x(t)+\Delta A^{1}(t) x(t)+\sum_{i=1}^{r} \Delta A^{2 i}(t) x\left(t-\tau_{i}(t)\right)+(b+\Delta b(t)) u(t), \\
y(t)=\left(C^{\prime}+\Delta C^{\prime}(t)\right) x(t),
\end{gathered}
$$

with an initial curve $\phi \in \mathbb{D}^{n}\left[t_{0}-\tau_{0}, t_{0}\right]$. Here, $A^{0}, \Delta A^{1}(t), \Delta A^{2 i}(t)(i=1, \ldots, r)$ are all real $n \times n$ matrices, where $r$ is a fixed positive integer; also, $A^{0}$ is a known constant matrix. Furthermore, $\Delta A^{1}(t)$ and $\Delta A^{2 i}(t)(i=1, \ldots, r)$ are uncertain coefficient matrices and may vary with $t \in$ $\left[t_{0}, \infty\right)$. Other variables are as follows: $u(t) \in \mathbb{R}$ is a control variable, $b \in \mathbb{R}^{n}$ is a known constant vector, and $\Delta b(t) \in \mathbb{R}^{n}$ is an uncertain coefficient vector which may vary with $t \in$ $\left[t_{0}, \infty\right) \cdot y(t) \in \mathbb{R}^{2}$ is an output variable, $C \in \mathbb{R}^{n \times 2}$ is a known constant matrix, and $\Delta C(t) \in \mathbb{R}^{n \times 2}$ is an uncertain coefficient matrix which may vary with $t \in\left[t_{0}, \infty\right)$.

In addition, all $\tau_{i}(t)(i=1, \ldots, r)$ are piecewise continuous functions and are uniformly bounded, that is, for nonnegative constant $\tau_{0}$ they satisfy

$$
0 \leq \tau_{i}(t) \leq \tau_{0} \quad(i=1, \ldots, r)
$$

for all $t \geq t_{0}$. The upper bound $\tau_{0}$ can be arbitrarily large and is not necessarily assumed to be known.

It is assumed that all entries of $\Delta A^{1}(t), \Delta A^{2 i}(t), \Delta b(t)$, and $\Delta C(t)$ are piecewise continuous functions and are uniformly bounded, that is, for nonnegative constant matrices $\Delta A^{10}, \Delta A^{2 i 0} \in \mathbb{R}^{n \times n}, \Delta C^{0} \in \mathbb{R}^{n \times 2}$, and for a nonnegative constant vector $\Delta b^{0} \in \mathbb{R}^{n}$, they satisfy

$$
\begin{array}{cc}
\left|\Delta A^{1}(t)\right| \leq \Delta A^{10}, & \left|\Delta A^{2 i}(t)\right| \leq \Delta A^{2 i 0}, \\
|\Delta b(t)| \leq \Delta b^{0}, & |\Delta C(t)| \leq \Delta C^{0}
\end{array}
$$

for all $t \geq t_{0}$. The upper bound of each entry can independently take an arbitrarily large value, but each is assumed to be known.

Assumption 3.1. Because the system must be controllable, we assume that the pair $\left(A^{0}, b\right)$ of the nominal system is a controllable pair and is in the controllable canonical form. Then $A^{0}$ 
and $b$ are given as follows:

$$
A^{0}=\left[\begin{array}{cccc}
0 & 1 & 0 & 0 \\
0 & \ddots & \ddots & 0 \\
0 & 0 & 0 & 1 \\
0 & 0 & 0 & 0
\end{array}\right], \quad b=\left[\begin{array}{l}
0 \\
\vdots \\
0 \\
1
\end{array}\right] .
$$

Assumption 3.2. Because the system must be observable, we assume that $C=\left(c_{1}, c_{2}\right) \in \mathbb{R}^{n \times 2}$ is given as follows:

$$
\begin{gathered}
c_{1}=(1,0, \ldots, 0)^{\prime}, \\
c_{2}=(0, \ldots, 0,1,0, \ldots, 0)^{\prime},
\end{gathered}
$$

where all entries of $C$ are equal to zero except that the first entry and the $k$ th entry of $c_{1}$ and $c_{2}$ are equal to 1 , respectively. $k$ has a strong relation to the configuration of uncertain entries and is defined in the subsequent discussion.

Considering a necessary and sufficient condition for linear uncertain systems to be observability invariant [8], we see that the observability of a given system might be lost without Assumption 3.2.

Next, we consider the following system:

$$
\dot{z}(t)=\left(A^{0}-L C^{\prime}\right) z(t)+L y(t)+b u(t)
$$

where $z(t) \in \mathbb{R}^{n}$ is an auxiliary state variable, and $L \in \mathbb{R}^{n \times 2}$ is a constant matrix. This is an observer in the most basic sense. Our objective is to find a controller for stabilizing the overall $2 n$-dimensional system consisting of (3.1) and (3.6). Let $e(t)$ be defined by

$$
e(t)=z(t)-x(t)
$$

Let $u(t)$ be given by

$$
u(t)=g^{\prime} z(t)=g^{\prime} e(t)+g^{\prime} x(t),
$$

where $g \in \mathbb{R}^{n}$ is a constant vector.

Definition 3.3. System (3.1) is said to be delay independently stabilizable if there exists a linear memoryless state feedback control $u(t)=g^{\prime} z(t)$ such that the equilibrium point $x=0$ of the resulting closed-loop system is uniformly and asymptotically stable for all admissible uncertain delays and uncertain parameters. 


\section{Preliminaries}

The $2 n$-dimensional system consisting of $x(t) \in \mathbb{R}^{n}$ and $e(t) \in \mathbb{R}^{n}$ is written as follows:

$$
\begin{aligned}
\dot{w}(t)= & {\left[\begin{array}{cc}
A^{0}-L C^{\prime} & 0 \\
0 & A^{0}+b g^{\prime}
\end{array}\right] w(t)+\left[\begin{array}{cc}
-\Delta b(t) g^{\prime} & -\Delta A^{1}(t)+L \Delta C^{\prime}(t)-\Delta b(t) g^{\prime} \\
b g^{\prime}+\Delta b(t) g^{\prime} & \Delta A^{1}(t)+\Delta b(t) g^{\prime}
\end{array}\right] w(t) } \\
& +\sum_{i=1}^{r}\left(\begin{array}{cc}
0 & -\Delta A^{2 i}(t) \\
0 & \Delta A^{2 i}(t)
\end{array}\right) w\left(t-\tau_{i}(t)\right),
\end{aligned}
$$

where $w(t)=\left(e^{\prime}(t), x^{\prime}(t)\right)^{\prime} \in \mathbb{R}^{2 n}$.

Because of Assumption 3.1, it is possible to choose $g \in \mathbb{R}^{n}$ such that all eigenvalues of $\left(A^{0}+b g^{\prime}\right)$ are real, negative, and distinct. Likewise, because of Assumption 3.2, it is possible to choose $L \in \mathbb{R}^{n \times 2}$ such that all eigenvalues of $\left(A^{0}-L C^{\prime}\right)$ are real, negative, and distinct. Let $g$ and $L$ be chosen in such a way. In addition, let $\lambda_{1}, \lambda_{2}, \ldots, \lambda_{n}$ and $\sigma_{1}, \sigma_{2}, \ldots, \sigma_{n}$ be the eigenvalues of $\left(A^{0}+b g^{\prime}\right)$ and $\left(A^{0}-L C^{\prime}\right)$, respectively. Let $T$ and $S$ be Vandermonde matrices constructed from $\lambda_{i}$ and $\sigma_{i}$, respectively, as follows:

$$
\begin{gathered}
T=\left[\begin{array}{cccccc}
1 & 1 & \ldots & \ldots & \ldots & 1 \\
\lambda_{1} & \lambda_{2} & \ldots & \ldots & \ldots & \lambda_{n} \\
\lambda_{1}{ }^{2} & \lambda_{2}{ }^{2} & \ldots & \ldots & \ldots & \lambda_{n}{ }^{2} \\
\vdots & \vdots & \vdots & \vdots & \vdots & \vdots \\
\lambda_{1}{ }^{n-1} & \lambda_{2}{ }^{n-1} & \ldots & \ldots & \ldots & \lambda_{n}{ }^{n-1}
\end{array}\right], \\
S=\left[\begin{array}{cc}
S^{1} & 0 \\
0 & S^{2}
\end{array}\right]
\end{gathered}
$$

where $S^{1}$ and $S^{2}$ are given by

$$
\begin{gathered}
S^{1}=\left[\begin{array}{ccccc}
\sigma_{1}^{k-2} & \sigma_{1}^{k-3} & \cdots & \sigma_{1} & 1 \\
\sigma_{2}^{k-2} & \sigma_{2}^{k-3} & \cdots & \sigma_{2} & 1 \\
\vdots & \vdots & \cdots & \vdots & \vdots \\
\sigma_{k-1}^{k-2} & \sigma_{k-1}^{k-3} & \cdots & \sigma_{k-1} & 1
\end{array}\right], \\
S^{2}=\left[\begin{array}{ccccc}
\sigma_{k}^{n-k} & \sigma_{k}^{n-k-1} & \cdots & \sigma_{k} & 1 \\
\sigma_{k+1}^{n-k} & \sigma_{k+1}^{n-k-1} & \cdots & \sigma_{k+1} & 1 \\
\vdots & \vdots & \cdots & \vdots & \vdots \\
\sigma_{n}^{n-k} & \sigma_{n}^{n-k-1} & \cdots & \sigma_{n} & 1
\end{array}\right] .
\end{gathered}
$$


$T$ and $S$ are well known to be nonsingular in view of the previous assumptions. Define $\Lambda$ and $\Sigma$ as follows:

$$
\begin{aligned}
& \Lambda=T^{-1}\left(A^{0}+b g^{\prime}\right) T=\operatorname{diag}\left(\lambda_{1}, \lambda_{2}, \ldots, \lambda_{n}\right), \\
& \Sigma=S\left(A^{0}-L C^{\prime}\right) S^{-1}=\operatorname{diag}\left(\sigma_{1}, \sigma_{2}, \ldots, \sigma_{n}\right) .
\end{aligned}
$$

Let $P^{1}, P^{2}$, and $P^{3}$ be defined as follows:

$$
\begin{aligned}
P^{1} & =\left[\begin{array}{cc}
\Sigma & 0 \\
0 & \Lambda
\end{array}\right], \\
P^{2} & =\left[\begin{array}{cc}
0 & |S| \Delta A^{30}|T| \\
\left|T^{-1}\right|\left|b g^{\prime}\right|\left|S^{-1}\right| & \left|T^{-1}\right| \Delta A^{30}|T|
\end{array}\right], \\
P^{3} & =\left[\begin{array}{cc}
|S| \Delta b^{0}\left|g^{\prime}\right|\left|S^{-1}\right| & |S|\left(|L| \Delta C^{0^{\prime}}+\Delta b^{0}\left|g^{\prime}\right|\right)|T| \\
\left|T^{-1}\right| \Delta b^{0}\left|g^{\prime}\right|\left|S^{-1}\right| & \left|T^{-1}\right| \Delta b^{0}\left|g^{\prime}\right||T|
\end{array}\right],
\end{aligned}
$$

where $\Delta A^{30}$ is given by

$$
\Delta A^{30}=\Delta A^{10}+\sum_{i=1}^{r} \Delta A^{2 i 0}
$$

In addition, let $P$ be defined by

$$
P=-P^{1}-P^{2}-P^{3}
$$

Here, we introduce the fundamental lemma which plays a crucial role to lead the main results.

Lemma 4.1 (see [6]). If there exist $T$ and $S$ which assure

$$
P \in \mathcal{M}
$$

then system (3.1) is delay independently stabilizable.

Note that our problem has been reduced to finding $T$ and $S$ that enable $P$ to satisfy condition (4.8). In the subsequent discussion, we consider the possibility of choosing $T$ and $S$ that assure $P \in \mathcal{M}$.

\section{Main Results}

First, we introduce a set of matrices $\Omega(k) \in \mathbb{R}^{(n+1) \times(n+1)}$ as follows. 
Definition 5.1. Let $k$ be an integer satisfying $0 \leq k \leq n$. For this $k$, let $\Omega(k)=\left\{D=\left(d_{i j}\right) \in\right.$ $\left.\mathbb{R}^{(n+1) \times(n+1)}\right\}$ be a set of matrices with the following properties:

(1) if $1 \leq j \leq k$, then $d_{i j}=0$, for $j+1 \leq i \leq 2 k-j$,

(2) if $k+1 \leq j \leq n+1$, then $d_{i j}=0$, for $2 k-j \leq i \leq j-1$.

Now, we state the main result.

Theorem 5.2. Construct a matrix $\Gamma \in \mathbb{R}^{(n+1) \times(n+1)}$ as

$$
\Gamma=\left[\begin{array}{cc}
\Delta c_{1}^{0^{\prime}} & 0 \\
\Delta A^{30} & \Delta b^{0}
\end{array}\right]
$$

by means of system parameters. If for fixed $k$,

$$
\Gamma \in \Omega(k), \quad \Delta c_{2}^{0}=0,
$$

then system (3.1) is delay independently stabilizable.

System (3.1) is said to have a triangular configuration if the system satisfies condition (5.2). A schematic view of the system having a triangular configuration is shown below. Here, $*$ indicates an uncertain entry not necessarily equal to zero

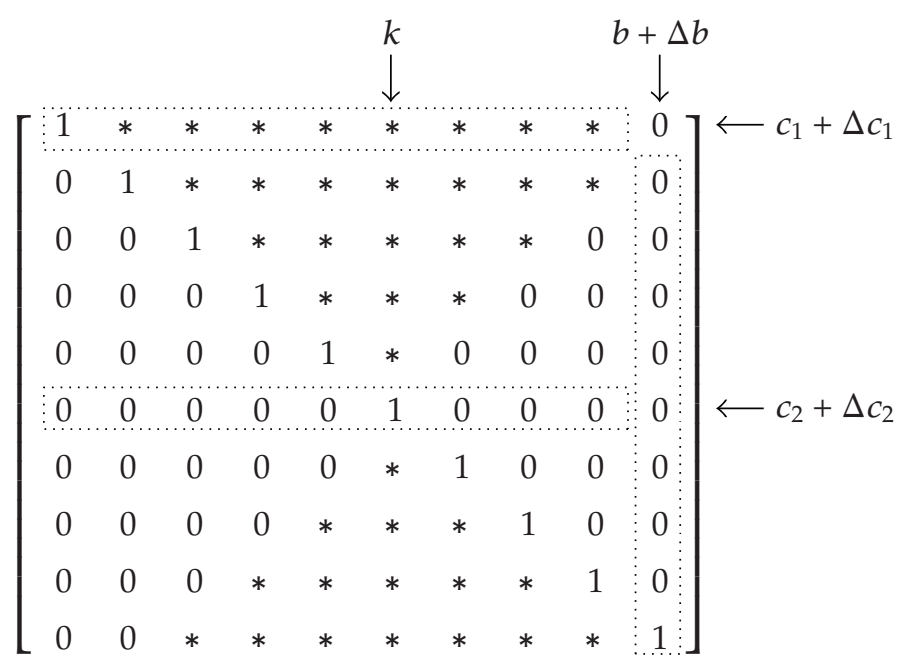

Proof of Theorem 5.2. According to Lemma 4.1, the existence of $T$ and $S$ which assure $P \in \mathcal{M}$ is shown in the rest of this section. Here, let $\mu$ be a positive number and let $\alpha_{i}(i=1, \ldots, n)$ be all negative numbers that are different from one another. Likewise, let $\beta_{i}(i=1, \ldots, n)$ be all negative numbers that are different from one another. Let $\mu$ be chosen larger than all 
upper bounds of uncertain elements $\Delta A^{30}, \Delta b^{0}$, and $\Delta c_{1}^{0}$. $\alpha_{i}$ and $\beta_{i}$ are used for distinguishing eigenvalues $\lambda_{i}$ and $\sigma_{i}$ from one another. Let $\lambda_{i}$ and $\sigma_{i}$ be chosen as follows:

$$
\begin{array}{cl}
\lambda_{i}=\alpha_{i} \mu^{-1} \in O(-1) & (i=1, \ldots, k-1), \\
\sigma_{i}=\beta_{i} \mu^{-1} \in O(-1) & (i=1, \ldots, k-1), \\
\lambda_{i}=\alpha_{i} \mu \in O(1) & (i=k, \ldots, n), \\
\sigma_{i}=\beta_{i} \mu \in O(1) & (i=k, \ldots, n) .
\end{array}
$$

Then, we can write $T$ and $S$ as follows:

$$
\begin{aligned}
& T:=\left[T^{1} \mid T^{2}\right] \\
& =\left[\begin{array}{c|c|}
\hline 0 & 0 \\
\vdots & \vdots \\
\hline-i+1 & i-1 \\
\hline \vdots & \vdots \\
\hline-n+1 & n-1
\end{array}\right], \\
& S^{1}=\left[\begin{array}{lll|l|l|l}
-k & & -k \\
+2 & \cdots & & \\
+j & \cdots & 0 \\
& & & & \\
\end{array}\right. \\
& S^{2}=\left[\begin{array}{ccc|c|c|c}
n & & n \\
-k & \cdots & & & \\
-j & \cdots & 0 \\
& & & &
\end{array}\right] \text {, }
\end{aligned}
$$

where $T^{1}$ and $T^{2}$ denote $n \times(k-1)$ and $n \times(n-k+1)$ matrices, respectively. In addition, $S^{1}$ and $S^{2}$ denote $(k-1) \times(k-1)$ and $(n-k+1) \times(n-k+1)$ matrices, respectively. In the above notation, $m$ and $m$ denote a row vector and a column vector, whose all entries are functions of $\mu$ of order $m$, respectively. For convenience, we adopt such notation for matrices in the subsequent discussion and neglect further explanation when it is clear. The notation of (5.5) means that all entries of the $i$ th row of $T^{1}$ and $T^{2}$ are functions of $\mu$ of order $(-i+1)$ and $(i-1)$, respectively. The notations of (5.6) and (5.7) mean that all entries of the $j$ th column of $S^{1}$ and $S^{2}$ are functions of $\mu$ of order $(-k+j+1)$ and $(n-k-j+1)$, respectively. 
Next, from the relations between the roots and the coefficients of the characteristic equations $\operatorname{det}\left(A^{0}+b g^{\prime}\right)$ and $\operatorname{det}\left(A^{0}-L C^{\prime}\right)$, we find that $g^{\prime}$ and $L$ have the following structure:

$$
\begin{aligned}
& g^{\prime}=\left[\begin{array}{c|c|c|c|c|c}
\hline n & & n & n \\
-2 k & \ldots & -k & & \\
-k & \ldots & 1 \\
+2 & & & & &
\end{array}\right], \\
& L=\left[\begin{array}{c|c}
\hline-1 & 0 \\
\vdots & \vdots \\
\hline-k+2 & 0 \\
\hline-k+1 & 1 \\
\hline 0 & 1 \\
0 & 2 \\
\vdots & \vdots \\
0 & n-k+1
\end{array}\right] .
\end{aligned}
$$

The notation of (5.8) means that the entry of the $j$ th column of $g^{\prime}$ is a function of $\mu$ of order $(n-2 k+j+1)$ if $j \leq k-1$, and of order $(n-j+1)$ if $j \geq k$. The notation of (5.9) means that the entry of the $i$ th row and the first column of $L$ is a function of $\mu$ of order $-i$ if $i \leq k-1$, and the entry of the $i$ th row and the second column of $L$ is of order $i-k+1$ if $i \geq k$.

Considering such structures of $T, S, g^{\prime}$ and $L$, it turns out from the careful calculation that each block matrix in (4.5) is further decomposed into four block matrices as follows:

$$
\begin{aligned}
\left|T^{-1}\right|\left|b g^{\prime}\right|\left|S^{-1}\right| & =\left[\begin{array}{l|l|l|}
\hline k-3 & 2 k-n-2 \\
\hline-k+1 & -n+2
\end{array}\right], \\
\left|T^{-1}\right| \Delta b^{0}\left|g^{\prime}\right|\left|S^{-1}\right| & =\left[\begin{array}{|l|l|}
\hline k-4 & 2 k-n-3 \\
\hline-k-2 & -n-1
\end{array}\right], \\
\left|T^{-1}\right| \Delta A^{30}|T| & =\left[\begin{array}{|l|l|}
\hline-2 & 2 k-4 \\
\hline-2 k+2 & 0 \\
\hline
\end{array}\right], \\
\left|T^{-1}\right| \Delta b^{0}\left|g^{\prime}\right||T| & =\left[\begin{array}{|l|l|}
\hline-2 & 2 k-4 \\
\hline-2 k & -2 \\
\hline
\end{array}\right],
\end{aligned}
$$


Mathematical Problems in Engineering

$$
\begin{aligned}
& |S| \Delta A^{30}|T|=\left[\begin{array}{c|c|}
\hline-k & k-2 \\
\hline n-2 k+1 & n-1 \\
\hline
\end{array}\right], \\
& |S||L| \Delta C^{0^{\prime}}|T|=\left[\begin{array}{cc|cc|cc}
\hline & -k & & \multicolumn{2}{|c|}{k-2} \\
\hline 0 & \cdots & 0 & 0 & \cdots & 0 \\
\vdots & \ddots & \vdots & \vdots & \ddots & \vdots \\
0 & \cdots & 0 & 0 & \cdots & 0
\end{array}\right], \\
& |S| \Delta b^{0}\left|g^{\prime}\right||T|=\left[\begin{array}{ccc|ccc}
\hline & -k & & \multicolumn{2}{|c|}{k-2} \\
\hline 0 & \cdots & 0 & 0 & \cdots & 0 \\
\vdots & \ddots & \vdots & \vdots & \ddots & \vdots \\
0 & \cdots & 0 & 0 & \cdots & 0
\end{array}\right], \\
& |S| \Delta b^{0}\left|g^{\prime}\right|\left|S^{-1}\right|=\left[\begin{array}{ccc|ccc}
\hline & -2 & & k-n-1 \\
\hline 0 & \cdots & 0 & 0 & \cdots & 0 \\
\vdots & \ddots & \vdots & \vdots & \ddots & \vdots \\
0 & \cdots & 0 & 0 & \cdots & 0
\end{array}\right] .
\end{aligned}
$$

In the above notation, all the entries of each block matrix are functions of $\mu$ of the same order. Now, let $P \in \mathbb{R}^{2 n \times 2 n}$ in (4.7) be decomposed into four block matrices as follows:

$$
P=\left[\begin{array}{ll}
P_{11} & P_{12} \\
P_{21} & P_{22}
\end{array}\right]
$$

where

$$
\begin{aligned}
& P_{11}=-\Sigma-|S| \Delta b^{0}\left|g^{\prime}\right|\left|S^{-1}\right| \\
& P_{12}=-|S| \Delta A^{30}|T|-|S||L| \Delta C^{0^{\prime}}|T|-|S| \Delta b^{0}\left|g^{\prime}\right||T|, \\
& P_{21}=-\left|T^{-1}\right|\left|b g^{\prime}\right|\left|S^{-1}\right|-\left|T^{-1}\right| \Delta b^{0}\left|g^{\prime}\right|\left|S^{-1}\right| \\
& P_{22}=-\Lambda-\left|T^{-1}\right| \Delta A^{30}|T|-\left|T^{-1}\right| \Delta b^{0}\left|g^{\prime}\right||T| .
\end{aligned}
$$


It is apparent that $P \in \mathcal{M}$ if and only if

$$
\begin{gathered}
P_{11} \in \mathcal{M}, \\
P_{22} \in \mathcal{M}, \\
P_{22}-P_{21} P_{11}^{-1} P_{12} \in \mathcal{M} .
\end{gathered}
$$

The following lemma shown in [9] is useful for verification of whether a given matrix is an M-matrix.

Lemma 5.3 (see [9]). Let $k$ be an integer satisfying $1<k<n$. Let $B \in \mathbb{R}^{n \times n}$ be a diagonal matrix whose every entry is positive, and let $C \in \mathbb{R}^{n \times n}$. Let $B$ and $C$ be decomposed into four block matrices as follows:

$$
B=\left(\begin{array}{l|l}
B_{11} & 0 \\
\hline 0 & B_{22}
\end{array}\right), \quad C=\left(\begin{array}{l|l}
C_{11} & C_{12} \\
\hline C_{21} & C_{22}
\end{array}\right) .
$$

Therein, $B_{11}$ and $B_{22}$ are $k \times k$ and $(n-k) \times(n-k)$ diagonal matrices, respectively. $C_{11}, C_{12}, C_{21}$, and $C_{22}$ are $k \times k, k \times(n-k),(n-k) \times k$, and $(n-k) \times(n-k)$ block matrices, respectively. Suppose that all the entries of each block matrix are functions of $\mu$ of the same order. Let all the entries of $B_{11}$ and $B_{22}$ belong to $O\left(b_{11}\right)$ and $O\left(b_{22}\right)$, respectively. Let all the entries of $C_{11}, C_{12}, C_{21}$, and $C_{22}$ belong to $O\left(c_{11}\right), O\left(c_{12}\right), O\left(c_{21}\right)$, and $O\left(c_{22}\right)$, respectively. For sufficiently large $\mu$, if

$$
\begin{gathered}
b_{11}>c_{11}, \\
b_{22}>c_{22}, \\
b_{11}>c_{12}-b_{22}+c_{21},
\end{gathered}
$$

then the matrix $A=B-|C|$ is an M-matrix.

Using Lemma 5.3, we can deduce whether the matrix whose entry represents the functional order is an $M$-matrix.

Taking into account the fact that $\Sigma$ is a diagonal matrix in which all diagonal entries belong to $O(-1)$ from the first to $(k-1)$ th entry or $O(1)$ from the $k$ th to $n$th entry, we see from (5.17) that $P_{11} \in \mathcal{M}$.

From (5.14)-(5.16), it follows that

$$
P_{12}=\left[\begin{array}{c|c|}
\hline-k & k-2 \\
\hline n-2 k+1 & n-1
\end{array}\right] .
$$


From (5.10) and (5.11), we have

$$
P_{21}=\left[\begin{array}{l|l|}
\hline k-3 & 2 k-n-2 \\
\hline-k+1 & -n+2
\end{array}\right] .
$$

From (5.12) and (5.13), it follows that

$$
P_{22}=-\Lambda-\left[\begin{array}{|c|c|}
\hline-2 & 2 k-4 \\
\hline-2 k+2 & 0
\end{array}\right] .
$$

Taking into account the fact that $\Lambda$ is a diagonal matrix in which all diagonal entries belong to $O(-1)$ from the first to $(k-1)$ th entry or $O(1)$ from the $k$ th to $n$th entry, we obtain

$$
\begin{gathered}
-1>-2, \\
1>0 \\
-1>2 k-4-1-2 k+2=-3 .
\end{gathered}
$$

According to Lemma 5.3, the inequalities (5.26) show that $P_{22} \in \mathcal{M}$. From (5.23) and (5.24), it follows that

$$
P_{21} P_{11}^{-1} P_{12}=\left[\begin{array}{|c|c|}
\hline-2 & 2 k-4 \\
\hline-2 k+2 & 0 \\
\hline
\end{array}\right] .
$$

Then, from (5.25) and (5.27), we have

$$
P_{22}-P_{21} P_{11}^{-1} P_{12}=-\Lambda-\left[\begin{array}{|l|l|}
\hline-2 & 2 k-4 \\
\hline-2 k+2 & 0 \\
\hline
\end{array}\right] .
$$

Hence, it is apparent from the inequalities (5.26) that $P_{22}-P_{21} P_{11}^{-1} P_{12} \in \mathcal{M}$. Taking into account the fact that all the conditions of (5.20) hold, we see that $P \in \mathcal{M}$.

Therefore, using Lemma 4.1, we can conclude that system (3.1) is delay independently stabilizable.

\section{Conclusions}

The stabilization problem of linear time-varying uncertain delay systems with limited measurable state variables was studied here. Each uncertain parameter and each delay under consideration may take arbitrarily large values. It was shown that if the uncertain entries enter the system matrices in a way to form a particular geometric pattern called a triangular 
configuration, then the system is stabilizable irrespectively of the given bounds of uncertain parameters and delays. The method that enables verification of whether the transformed system satisfies the $M$-matrix stability condition was provided here. Moreover, it was shown that the constructed variable transformation enables the system to satisfy the $M$-matrix stability condition. The obtained conditions have a strong similarity to the ones called an antisymmetric stepwise configuration by Wei [4]. To develop the conditions obtained here into the ones of antisymmetric stepwise configurations is a problem to be considered next.

\section{Acknowledgment}

This research was partially supported by the Ministry of Education, Science, Sports and Culture, Grant-in-Aid for Young Scientists (Start-up), 20860040.

\section{References}

[1] M. S. Mahmoud and N. F. Al-Muthairi, "Quadratic stabilization of continuous time systems with statedelay and norm-bounded time-varying uncertainties," IEEE Transaction on Automatic Control, vol. 39, no. 10, pp. 2135-2139, 1994.

[2] S.-I. Niculescu, " $H_{\infty}$ memoryless control with an $\alpha$-stability constraint for time-delay systems: an LMI approach," IEEE Transaction on Automatic Control, vol. 43, no. 5, pp. 739-743, 1998.

[3] E. Fridman and U. Shaked, "Parameter dependent stability and stabilization of uncertain time-delay systems," IEEE Transaction on Automatic Control, vol. 48, no. 5, pp. 861-866, 2003.

[4] K. H. Wei, "Quadratic stabilizability of linear systems with structural independent time-varying uncertainties," IEEE Transaction on Automatic Control, vol. 35, no. 3, pp. 268-277, 1990.

[5] T. Amemiya and G. Leitmann, "A method for designing a stabilizing control for a class of uncertain linear delay systems," Dynamics and Control, vol. 4, no. 2, pp. 147-167, 1994.

[6] T. Amemiya, "A stabilizing control for a class of uncertain delay systems with limited measurable state variables," Dynamics and Control, vol. 7, no. 3, pp. 235-262, 1997.

[7] T. Amemiya, "Triangular configuration of uncertain systems stabilizable by means of feedback controller," Applied Mathematics and Computation, vol. 120, no. 1-3, pp. 45-54, 2001.

[8] T. Hashimoto and T. Amemiya, "Controllability and observability invariance of linear time-invariant uncertain systems," in Proceedings of the International Automatic Control Conference, Tainan, Taiwan, 2008, CD-ROM FA06-4.

[9] T. Hashimoto and T. Amemiya, "A note on a criterion for M-matrix," Computational Mathematics and Modeling, vol. 20, no. 3, pp. 318-325, 2009. 


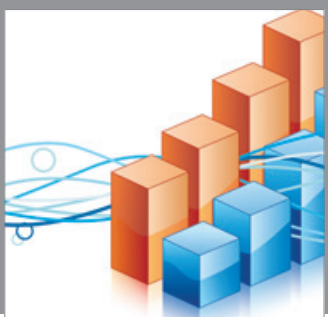

Advances in

Operations Research

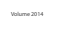

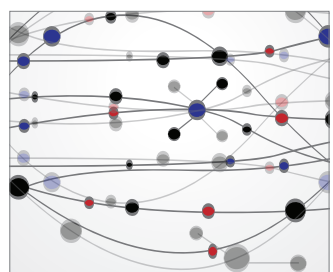

\section{The Scientific} World Journal
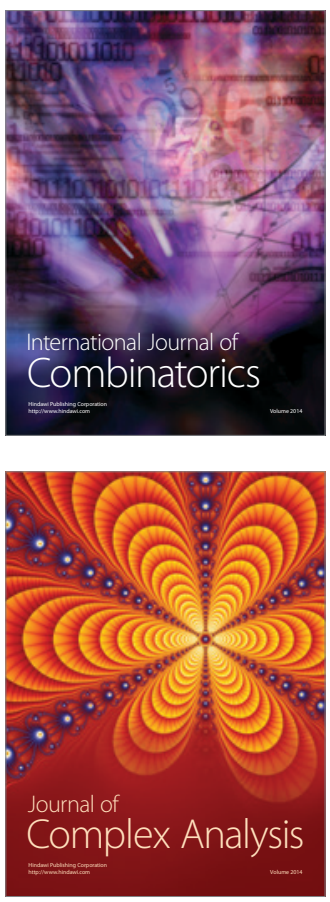

International Journal of

Mathematics and

Mathematical

Sciences
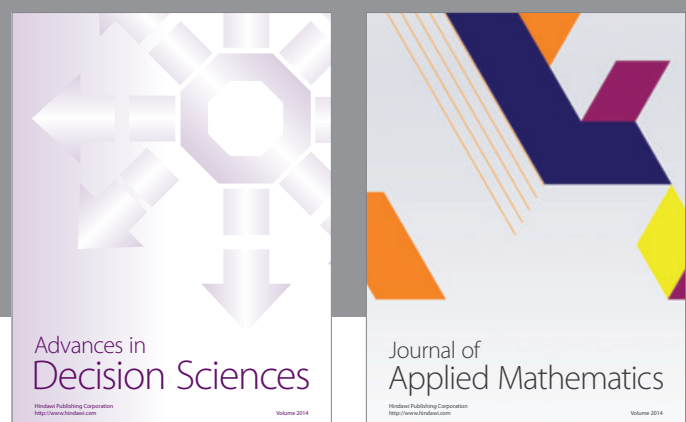

Journal of

Applied Mathematics
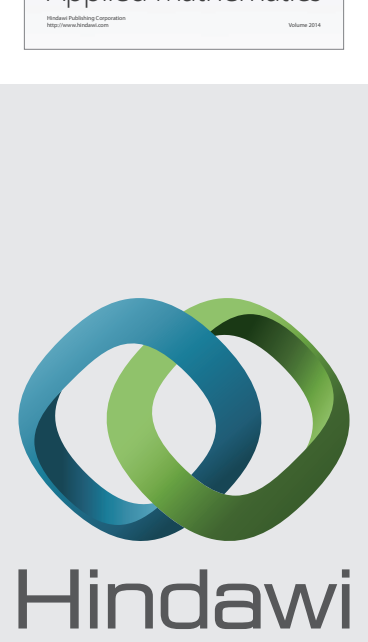

Submit your manuscripts at http://www.hindawi.com
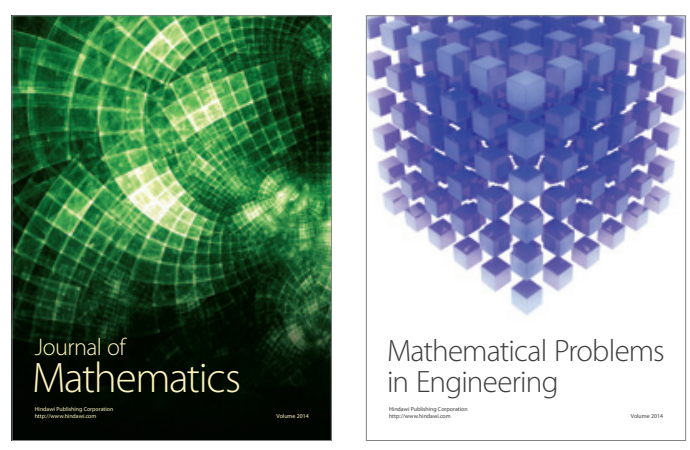

Mathematical Problems in Engineering
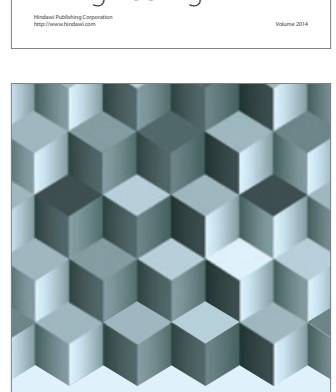

Journal of

Function Spaces
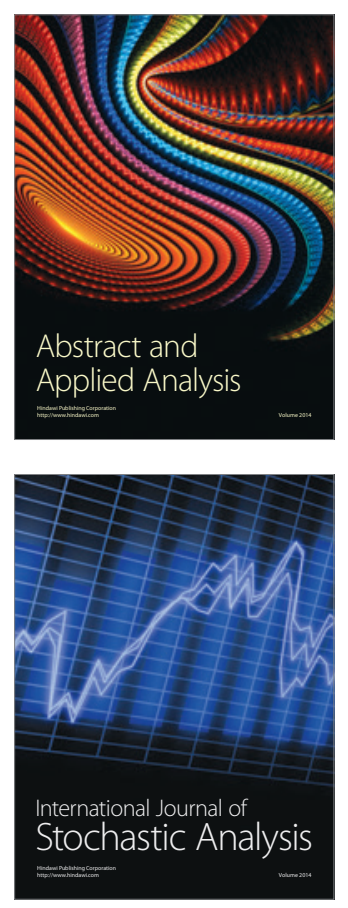

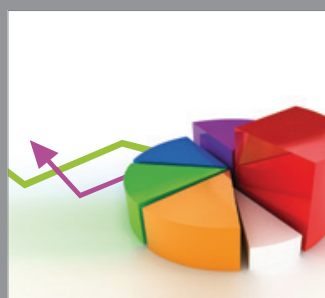

ournal of

Probability and Statistics

Promensencen
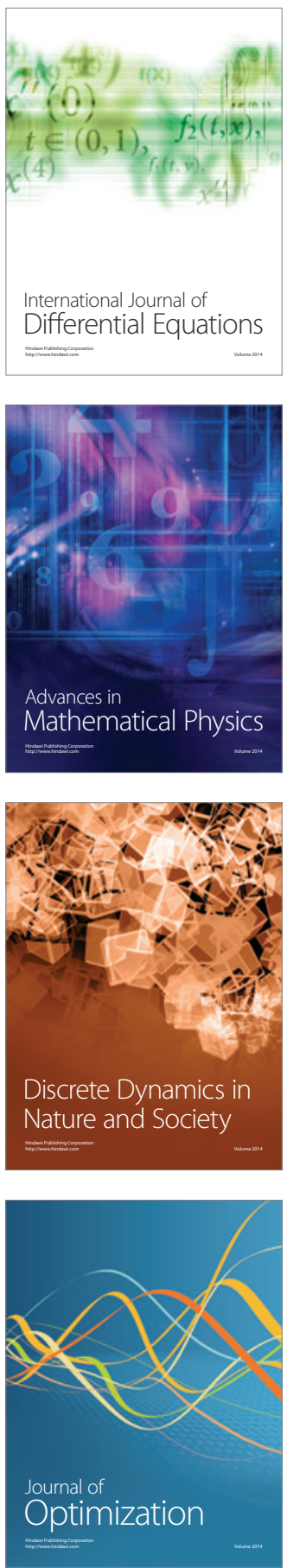